\title{
Viral vector-based vaccines against SARS-CoV-2
}

\author{
Kenneth Lundstrom ${ }^{*}$ (1) \\ PanTherapeutics, CH1095 Lutry, Switzerland \\ *Correspondence: Kenneth Lundstrom, PanTherapeutics, CH1095 Lutry, Switzerland. lundstromkenneth@gmail.com \\ Academic Editor: Wangxue Chen, National Research Council Canada, Canada \\ Received: August 19, 2021 Accepted: October 18, 2021 Published: October 31, 2021
}

Cite this article: Lundstrom K. Viral vector-based vaccines against SARS-CoV-2. Explor Immunol. 2021;1:295-308. https://doi. org/10.37349/ei.2021.00020

\begin{abstract}
Viral vectors have been frequently applied for vaccine development. It has also been the case for vaccines against severe acute respiratory syndrome coronavirus 2 (SARS-CoV-2) to tackle the coronavirus disease 2019 (COVID-19) pandemic. A multitude of different viral vectors have been mainly targeting the SARS-CoV-2 spike $(\mathrm{S})$ protein as antigen. Intramuscular injection has been most commonly used, but also intranasal administration has been tested. Adenovirus vector-based vaccines are the most advanced with several vaccines receiving Emergency Use Authorization (EUA). The simian ChAdOx1 nCoV-19 vaccine applied as a prime-boost regimen has provided $62.1-90 \%$ vaccine efficacy in clinical trials. The Ad26.COV2.S vaccine requires only one immunization to provide protection against SARS-CoV-2. The rAd26-S/rAd5-S vaccine utilizes the Ad26 serotype for the prime immunization followed by a boost with the Ad5 serotype resulting in $91.2 \%$ vaccine efficacy. All adenovirus-based vaccines are used for mass vaccinations. Moreover, vaccine candidates based on vaccinia virus and lentivirus vectors have been subjected to clinical evaluation. Among self-replicating RNA viruses, vaccine vectors based on measles virus, rhabdoviruses, and alphaviruses have been engineered and tested in clinical trials. In addition to the intramuscular route of administration vaccine candidates based on influenza viruses and adenoviruses have been subjected to intranasal delivery showing antibody responses and protection against SARS-CoV- 2 challenges in animal models. The detection of novel more transmissible and pathogenic SARS-CoV-2 variants added concerns about the vaccine efficacy and needs to be monitored. Moreover, the cause of recently documented rare cases of vaccine-induced immune thrombotic thrombocytopenia (VITT) must be investigated.
\end{abstract}

\section{Keywords}

Viral vectors, vaccines, SARS-CoV-2, COVID-19, clinical trials, protection

\section{Introduction}

The current coronavirus disease 2019 (COVID-19) pandemic has triggered an intensive race to develop numerous vaccine candidates targeting severe acute respiratory syndrome coronavirus 2 (SARS-CoV-2) [1]. No stones were left unturned as all potential approaches such as vaccines based on inactivated and attenuated viruses, protein subunits, nucleic acids and viral vectors have been evaluated [2]. The first COVID-19 vaccine 
to receive Emergency Use Authorization (EUA) was the BNT162b2 mRNA-based vaccine, which has now been subjected to mass vaccinations around the world [3]. It was quickly followed by another mRNA-based vaccine, mRNA-1273 [4]. Vaccines based on inactivated viruses have also recently been approved in China [5]. Moreover, for protein subunit vaccine candidates [6] and DNA-based vaccine candidates [7], promising results have been obtained in clinical trials. Viral vectors have previously been used frequently for vaccine development [8] and COVID-19 vaccines are no exception. This review focuses uniquely on viral vector-based COVID-19 vaccines, describing their specific features, preclinical studies in animal models, and clinical evaluation in humans. The effect on the efficacy of viral vector-based vaccines by the recently discovered SARS-CoV-2 variants and vaccine-induced immune thrombotic thrombocytopenia (VITT) are also discussed.

\section{Viral vector-based COVID-19 vaccines}

Various viral delivery vectors based on adenoviruses, vaccinia viruses, lentiviruses, measles virus, rhabdoviruses, alphaviruses and influenza viruses have been engineered for SARS-CoV-2 vaccines and subjected to preclinical studies in animal models and clinical evaluation in healthy volunteers (Table 1). The common feature of the vaccine candidates based on viral vectors is the utilization of the SARS-CoV-2 spike (S) protein as the antigen although in some applications the SARS-CoV-2 nucleocapsid (N) protein has also been targeted. The route of administration has in the majority of applications focused on intramuscular administration. However, as SARS-CoV-2 infections occur through the respiratory tract [9], intranasal delivery has been considered as an attractive alternative. In this context, both adenovirus- and influenza virus-based vectors have been developed.

Table 1. Preclinical and clinical studies for viral vector-based COVID-19 vaccines

\begin{tabular}{|c|c|c|c|}
\hline Vector & Construct & Findings & Ref \\
\hline ChAdOx1 & ChAdOx1 nCoV-19 & Protection against SARS-CoV-2 in macaques & [11] \\
\hline ChAdOx1 & ChAdOx1 nCoV-19 & Protection after intranasal injection in mice & [11] \\
\hline ChAdOx1 & ChAdOx1 nCoV-19 & Phase I/II: safety, strong immune responses & [12] \\
\hline ChAdOx1 & ChAdOx1 nCoV-19 & Phase II/III: high Ab titers in all age groups & [13] \\
\hline ChAdOx1 & ChAdOx1 nCoV-19 & Phase III: > 30,000 healthy volunteers & [14] \\
\hline ChAdOx1 & ChAdOx1 nCoV-19 & Phase III: $62.1-90.0 \%$ vaccine efficacy & [15] \\
\hline ChAdOx1 & ChAdOx1 nCoV-19 & EUA in the UK in December 2020 & [16] \\
\hline Ad26 & Ad26.COV2.S & Protection of hamsters after single injection & [18] \\
\hline Ad26 & Ad26.COV2.S & Protection of macaques after single injection & [19] \\
\hline Ad26 & Ad26.COV2.S & Phase I: single injection > immune response & [20] \\
\hline Ad26 & Ad26.COV2.S & Phase I/II: strong immune response & [21] \\
\hline Ad26 & Ad26.COV2.S & Phase III: > 60,000 healthy volunteers & [22] \\
\hline Ad26 & Ad26.COV2.S & EUA by the FDA in February 2021 & [23] \\
\hline Ad26/Ad5 & rAd26-S/rAd5-S & $100 \%$ protection in mice, hamsters \& primates & [24] \\
\hline Ad26/Ad5 & rAd26-S/rAd5-S & Phase I/II: safety, strong immune response & [25] \\
\hline Ad26/Ad5 & rAd26-S/rAd5-S & Phase III: $91.6 \%$ vaccine efficacy & [26] \\
\hline Ad26/Ad5 & rAd26-S/rAd5-S & EUA in Russia in July 2020 & [27] \\
\hline Ad5 & Ad5-S-nb2 & SARS-CoV-2 protection in mice \& ferrets & [28] \\
\hline Ad5 & Ad5-S.nb2 & SARS-CoV-2 protection in macaques & [29] \\
\hline Ad5 & Ad5-S.nb2 & Phase I: binding and neutralization Abs & [30] \\
\hline Ad5 & Ad5-S.nb2 & Phase II: Abs dependent on age, pre-existing Ad5 & [31] \\
\hline Ad5 & Ad5-S.nb2 & $90.1 \%$ efficacy of preventing severe COVID-19 & [32] \\
\hline Ad5 & Ad5-S.nb2 & EUA in China in February 2021 & [33] \\
\hline Ad5 & Ad5-S nb2 & Protection in macaques after i.n. administration & [29] \\
\hline Ad5 & AdCOVID-RBD & Systemic \& mucosal immunity after i.n. delivery & [59] \\
\hline Ad5 & VXA-COV2-1 & Strong immune response after i.n. delivery in mice & [66] \\
\hline Ad5 & VXA-COV2-1 & Phase I: oral delivery, strong immunogenicity & [69] \\
\hline GRAd & GRAd-COV2 & Robust immune responses in mice \& macaques & [34] \\
\hline Grad & GRAd-COV2 & Phase I: study in progress & [35] \\
\hline
\end{tabular}


Table 1. Preclinical and clinical studies for viral vector-based COVID-19 vaccines (continued)

\begin{tabular}{|c|c|c|c|}
\hline Vector & Construct & Findings & Ref \\
\hline ChAd68 & ChAdV68 + SAM & Phase I: study in progress & [37] \\
\hline AAVCOVID-1 & AAV2-CoV-2 S & Protection in primates after single administration & [38] \\
\hline MVA & MVA-CoV-2 S/N & Humoral \& cellular immune responses in mice & [40] \\
\hline MVA & MVA-COV-2 S & Protection in mice & [41] \\
\hline MVA & MVA-COV-2 & Phase I: study in progress & [42] \\
\hline MVA & MVA-COV-2 & Phase I: study in progress & [43] \\
\hline NDV & NDV-SARS-CoV-2 S & Protection in mice and hamsters & [45] \\
\hline NDV & NDV-SARS-CoV-2 S & Protection in mice & [46] \\
\hline NDV & NDV-HXP-S & Phase I/II: study in progress & [47] \\
\hline MV & MV-SARS-CoV-2-S & Th1-biased and T-cell immune responses in mice & [49] \\
\hline MV & MV-SARS-CoV-2-S & Phase I: weak immunogenicity, trial discontinued & [51] \\
\hline VSV & VSV-SARS-CoV-2-S & Protection against SARS-CoV-2 pathogenesis in mice & [52] \\
\hline VSV & VSV-SARS-CoV-2-S & Phase I: weak immunogenicity, trial discontinued & [53] \\
\hline VSV & VSV- $\Delta G$ & Protection against SARS-CoV-2 in hamsters & [55] \\
\hline VSV & VSV- $\Delta G$ & Phase I/II: study in progress & [56] \\
\hline VEE RNA & VEE-SARS-CoV-2 S & Th1-biased immune response in mice & [58] \\
\hline VEE RNA & VEE-SARS-CoV-2 S & Phase I: dose-escalation study in progress & [59] \\
\hline LV & LV-SARS-COV-2-S & Reduced viral load after i.n. delivery in mice & [61] \\
\hline LV & LV-SMENP + CTL & Phase I/II: s.c. delivery of LV-DCs. i.v. CTLs & [62] \\
\hline Influenza & $\Delta \mathrm{NA}(\mathrm{RBD})$-Flu & Single i.n. dose: strong immune response & [63] \\
\hline Influenza & IFV-COV-2 S RBD & Phase I: registered trial for intranasal spray & [64] \\
\hline Influenza & IFV-COV-2 S RBD & Phase II: registered trial for intranasal spray & {$[65]$} \\
\hline
\end{tabular}

AAV: adeno-associated virus; Ab: antibodies; CTLs: cytotoxic T cell lymphocytes; DCs: dendritic cells; FDA: Food and Drug Administration; GRAd: gorilla adenovirus; i.n.: intranasal; IFV: influenza virus; i.v.: intravenous; LV: lentivirus; LV-SMENP: lentivirus vector expressing SARS-CoV-2 minigene; MV: measles virus; MVA: modified vaccinia virus Ankara; NDV: Newcastle disease virus; RBD: receptor binding domain; SAM: self-amplifying mRNA: s.c.: subcutaneous; VEE: Venezuelan equine encephalitis virus; VSV: vesicular stomatitis virus; VSV- $\Delta \mathrm{G}$ : VSV G protein

\section{Adenovirus and adeno-associated virus vector-based vaccines}

Adenovirus vectors have a long tradition as gene transfer and vaccine vectors and particularly the second and third generation adenovirus vectors have demonstrated high safety levels and good delivery efficacy [10]. The codon-optimized SARS-CoV-2 S protein has been utilized as the common antigen although, different strategies related to vector engineering have been applied. In one approach, the simian adenovirus vector ChAdOx1 was utilized to avoid any pre-existing adenovirus immunity in humans. The ChAdOx1 nCoV-19 vaccine candidate showed protection in immunized rhesus macaques [11]. The positive findings from preclinical studies in rodents and non-human primates supported the transfer to clinical trials with the ChAdOx1 nCoV-19 vaccine candidate. Good safety and both humoral and cellular immune responses were obtained in a phase I/II clinical trial [12]. Furthermore, interim results from a phase II/III study showed more frequent adverse events in younger individuals, but the elicited neutralizing antibody titers were similar for all age groups (18-15 years, 56-69 years, and 70 years and older) [13]. Additionally, phase III clinical evaluation in more than 30,000 volunteers have been conducted [14]. Interim phase III results from the UK, Brazil, and South Africa showed good vaccination safety and $62.1 \%$ vaccine efficacy after two vaccinations with $5 \times 10^{10}$ ChAdOx $1 \mathrm{nCoV}-19$ particles and up to $90 \%$ in individuals receiving a prime dose of $2.2 \times 10^{10}$ particles and a boost of $5.5 \times 10^{10}$ particles [15]. The ChAdOx1 nCoV-19 vaccine received an EUA in the UK in December 2020 [16]. In contrast to the ChAdOx1 nCoV-19 vaccine, the Ad26.COV2.S vaccine is based on the human Ad26 serotype expressing the prefusion-stabilized SARS-CoV-2 S protein, and requires only one immunization [17]. This was confirmed in hamsters, where a single injection of Ad26.COV2.S elicited neutralizing antibodies and protected the animals from SARS-CoV-2-associated pneumonia and death [18]. Moreover, a single immunization of macaques elicited strong neutralizing antibody responses and provided protection against SARS-CoV-2 challenges [19]. In the context of clinical trials, a single administration of Ad26.COV2.S elicited rapid binding, neutralization antibody responses and cellular immune responses in a phase I study in 25 healthy volunteers [20]. Moreover, 
1,045 healthy volunteers were vaccinated with a single dose of $1 \times 10^{10}$ or $5 \times 10^{10} \mathrm{Ad} 26$.COV2.S particles in a phase I/II study showing good safety and strong immune responses [21]. The Ad26.COV2.S vaccine has been subjected to large phase III clinical trials with 60,000 participants [22] and received an EUA by the FDA in February 2021 [23].

As mentioned earlier, simian adenovirus vectors have been used for SARS-CoV-2 vaccine development to address any potential pre-existing immunity against human adenoviruses in the population [11]. However, as the current adenovirus-based vaccines except for Ad26.COV2.S [18] require a prime-boost regimen, neutralizing antibodies against adenoviruses might reduce the efficacy of a second or a third immunization with the same adenovirus serotype. For this reason, a strategy of prime vaccination with an Ad26 serotype vector expressing the SARS-CoV-2 S protein followed by a booster vaccination with another adenovirus serotype, the Ad5 expressing the SARS-CoV-2 S protein, was evaluated.

In preclinical studies the rAd26-S/rAd5-S vaccine candidate showed $100 \%$ protection in transgenic mice, hamsters, and primates [24]. Moreover, good safety, mild adverse events, and robust immune responses were observed in a phase I/II clinical trial [25]. Interim results from a phase III study with the rAd26-S/rAd5-S vaccine showed good tolerability and $91.6 \%$ vaccine efficacy [26]. The rAd26-S/rAd5-S (Sputnik V) vaccine received an EUA in Russia already in July 2020 although only preliminary vaccine evaluation had been conducted in 76 volunteers [27].

A third generation Ad5 serotype vector expressing the SARS-CoV-2 S protein (Ad5-S-nb2) was intramuscularly administered into mice and ferrets, which resulted in protection against challenges with SARS-CoV-2 [28]. Moreover, the Ad5-S-nb2 vaccine provided projection against SARS-CoV-2 in rhesus macaques [29]. In the case of clinical trials, a single dose of Ad5-S-nb2 induced both binding and neutralizing antibodies in healthy volunteers [30]. However, the level of response depended on pre-existing Ad5 antibodies and the age of the vaccinated person [31]. Interim results from a phase III trial indicated that a single dose of the Ad5-S-nb2 vaccine showed an overall efficacy of $65.3 \%$ of preventing all symptomatic COVID-19 disease 28 days post-vaccination [32]. Moreover, Ad5-S-nb2 showed a 90.1\% efficacy of preventing severe COVID-19 disease 28 days post-immunization. The Ad5-S-nb2 received an EUA in February 2021 in China [33] and further in Mexico, Pakistan, and Hungary.

The gorilla adenovirus GRAd has been used for the expression of the prefusion stabilized SARS-CoV-2 S protein [34]. The GRAd-COV2 vaccine candidate elicited robust immunogenicity in both mice and macaques. The functional antibodies neutralized SARS-CoV-2 infection, blocked SARS-CoV-2 S protein binding to angiotensin-converting enzyme 2 (ACE2) and generated robust Thelper 1(Th1)-dominated cellular responses. The GRAd-COV2 vaccine candidate is currently in phase I evaluation [35]. In another vaccine approach, the chimpanzee adenovirus serotype 68 (ChAdV68) [36] was combined in a prime-boost regimen with a SAM expressing the SARS-CoV-2 S protein and T-cell epitopes from the SARS-CoV-2 N protein. A dose-escalation phase I clinical trial with a ChAdV68 prime vaccination and SAM boost vaccination is in progress [37].

Recently, the AAV vector-based vaccine candidate AAVCOVID-1 was introduced [38]. The SARS-CoV-2 S gene was expressed from an AAV2 inverted terminal repeat (ITR) with an AAVrh32.33 capsid, showing potent immunogenicity in mice and non-human primates. Moreover, a single immunization provided complete protection in macaques challenged with SARS-CoV-2. Neutralizing antibodies were sustained for a year. Neither pre-existing immunity against AAVCOVID-1 in humans nor cross-reactivity to common AAV vectors used in gene therapy were detected. Single dose administration, high yield manufacturing and stability for one month at room temperature make the AAV-based approach attractive for potential global use once efficacy has been confirmed in clinical trials.

\section{Vaccinia virus vector-based vaccines}

Poxviruses, especially vaccinia viruses and the MVA strain, have been regularly applied for vaccine development [39]. In the context of COVID-19 vaccines, a synthetic MVA-based vaccine platform has been engineered for co-expression of SARS-CoV-2 $\mathrm{S}$ and $\mathrm{N}$ proteins [40]. Mice immunized with the synthetic MVA (sMVA) vector induced robust SARS-CoV-2 antigen-specific humoral and cellular immune 
responses. In another study, mice subjected to a prime-boost regimen with either DNA/MVA-COV2-S or MVA-COV2-S/MVA-COV2-S showed broad SARS-CoV-2 S-specific CD4+ and CD8 ${ }^{+}$T-cell responses and also induced high immunoglobulin G (IgG) titers [41]. Immunization prevented viral replication in mouse lung and protected humanized K18-hACE2 mice from SARS-CoV-2 challenges. MVA-based vaccines have been subjected to the first-in-human phase I study [42]. Safety and tolerability are evaluated in healthy volunteers receiving two doses of either $1 \times 10^{7}$ or $1 \times 10^{8} \mathrm{IU}$ of MVA-SARS-COV-2 S. In another phase I study, a synthetic MVA vector expressing different regions of the SARS-CoV-2 genome is administered at doses of $1 \times 10^{7}, 1 \times 10^{8}$, and $2.5 \times 10^{8}$ pfu to 129 healthy volunteers for safety and tolerability analysis [43].

\section{Orthoavulavirus-based vaccines}

NDV vectors have previously been confirmed as attractive delivery vehicles in oncology [44]. In the context of COVID-19, application of a chimeric NDV vector generated stable expression of the membrane anchored SARS-CoV-2 S protein [45]. Immunization of mice and hamsters elicited robust binding and neutralizing antibodies and provided protection against SARS-CoV-2. Moreover, expression of wildtype and membraneanchored forms of the SARS-CoV-2 S protein from an NDV vector induced strong antibody responses and protected mice from SARS-CoV-2 infections [46]. A phase I/II clinical trial in healthy volunteers receiving the NDV-SARS-CoV-2 S vector (NDV-HXP-S) has started [47].

\section{Self-amplifying RNA virus vector-based vaccines}

Self-amplifying RNA viruses such as alphaviruses, flaviviruses, measles viruses, and rhabdoviruses, are the building blocks for the SAM technology [48]. The special feature of SAM comprises of the non-structural protein (nsPs) genes encoding for the replicase complex in alphaviruses and flaviviruses responsible for an estimated 200,000-fold amplification of viral RNA in infected host cells. In contrast, the RNA-dependent RNA polymerase (RdRp) is located in the structural genes of MV and rhabdoviruses, providing self-replication of viral RNA. For this reason, the SAM technology became an attractive alternative for vaccine development. Among self-replicating RNA viruses, MV vectors have been engineered for the expression of the full-length SARS-CoV-2 S protein [49]. A prime-boost immunization of mice with MV-SARS-CoV-2-S elicited high levels of effective Th1-biased antibody and T-cell responses. Protective S-specific IgG antibodies and multifunctional $\mathrm{CD}^{+}$and $\mathrm{CD}^{+}{ }^{+} \mathrm{T}$-cell responses were also detected. The MV-SARS-CoV-2-S vaccine candidate TMV-083 was evaluated in 90 volunteers in a phase I clinical trial [50]. Unfortunately, initial findings were disappointing as the immune responses in vaccinated volunteers were weaker than detected in convalescent COVID-19 patients, leading to the termination of the trial [51]. In another approach, a replication-competent VSV vector was engineered to express the SARS-CoV-2 S protein [52]. Immunization of BALB/c mice generated neutralizing antibody responses and provided protection from SARS-CoV-2-related pathogenesis. The VSV-SARS-CoV-2 $S$ vaccine candidate V590 has been subjected to a phase I trial to evaluate its safety and tolerability in 252 volunteers [53]. Despite good tolerability the trial was discontinued as the immune responses in vaccinated individuals were weaker than those seen in convalescent COVID-19 patients [54].

In another approach, the $G$ protein on the surface of replication-competent VSV particles was replaced by the SARS-CoV-2 S protein resulting in the chimeric VSV- $\Delta \mathrm{G}$ vector [55]. A single dose of $5 \times 10^{6} \mathrm{pfu}$ of VSV- $\Delta G$ elicited potent neutralizing antibodies and protected golden Syrian hamsters from challenges with SARS-CoV-2. Neither lung damage nor presence of viral load were detected in immunized hamsters. The VSV- $\Delta \mathrm{G}$ vaccine has entered a phase I/II clinical evaluation in 18-55-year-old volunteers receiving a single dose of $5 \times 10^{5}, 5 \times 10^{6}$ and $5 \times 10^{7}$ pfu of VSV- $\Delta G$ in part one of the study [56]. The focus in the second part will be on elderly volunteers, who will receive a single injection as in part one or two injections of $5 \times 10^{5} \mathrm{pfu}$ at 28 days interval. While the study was on-going, the EUA for the BNT162b2 mRNA vaccine was received, which resulted in an ethical and executional dilemma concerning the placebo arm of the phase I/II trial with the VSV- $\Delta$ G vaccine [57]. It was concluded that the placebo arm was critical for study quality, and it was decided that a follow-up prior to unblinding of 56 days provided a reasonable balance between ethics and execution. The individuals who received placebo were offered either an approved vaccine outside the study or to re-consent to the study with a 1:3 chance to receive placebo. 
An alternative approach based on SAM technology relates to the application of alphavirus RNA replicons. For instance, the Venezuelan equine encephalitis virus (VEE) RNA replicon expressing the prefusion-stabilized SARS-CoV-2 S protein was encapsulated in lipid nanoparticles (LNPs) [58]. Immunization of BALB/c mice with LNP-SARS-CoV-2 S RNA elicited dose-dependent SARS-CoV-2-specific antibody responses and neutralization of virus. The Th1-biased antibody responses were superior to the ones observed in convalescent COVID-19 patients. A phase I dose-escalation study of 0.1 to $1.0 \mu \mathrm{g}$ of LNP-SARS-CoV-2 S RNA is currently in progress in healthy volunteers to assess the safety of the vaccine candidate [59].

\section{Intranasal and oral delivery of viral vector-based vaccines}

In addition to conventional intramuscular vaccine administration, oral and nasal delivery approaches have been evaluated due to the potentially broad immune responses in the nasal mucosa, where SARS-CoV-2 infection occurs and the possibility to block both infection and transmission of SARS-CoV-2. In this context, intranasal administration of $1 \times 10^{10} \mathrm{Ad} 5-\mathrm{S} \mathrm{nb} 2$ particles provided protection of macaques [29]. Moreover, Ad5 expressing the receptor binding domain (RBD) of the SARS-CoV-2 S protein elicited strong RBD-specific IgA responses and $\mathrm{CD}^{+}{ }^{+}$and $\mathrm{CD} 8{ }^{+} \mathrm{T}$-cell responses with Th1-like cytokine profiles after intranasal administration in mice [60].

In another approach, a lentivirus vector expressing the full-length SARS-CoV-2 S protein was evaluated in ACE2-humanized mice [61]. Although robust immune responses were elicited, only partial protection against SARS-CoV-2 was achieved after systemic lentivirus administration. However, intranasal administration generated strong immune responses in the respiratory tract, a significant decrease in viral load in the lung, and reduced local inflammation. Studies in hamsters demonstrated prevention of deleterious lung injury. In the context of lentiviruses, although not based on intranasal administration, the LV-SMENP-DC vaccine utilizes modified DCs transduced with LV-SMENP and immunomodulatory genes [62]. In a phase I/II clinical trial volunteers will receive subcutaneously $5 \times 10^{6}$ lentivirus transduced DCs and $1 \times 10^{8}$ antigen specific CTLs for the evaluation of vaccine safety and efficacy.

Influenza virus vectors have been engineered by replacing the neuraminidase (NA) gene with a membrane-anchored form of the RBD of the SARS-CoV-2 S protein [63]. Robust neutralizing antibody responses against SARS-CoV-2, equivalent to levels observed in convalescent COVID-19 patients, were obtained after a single intranasal dose of the $\triangle N A(R B D)$-Flu vaccine. The influenza virus-based vaccine candidate has further been subjected to registration of phase I [64] and phase II [65] clinical trials in China for the delivery as an intranasal spray.

In the context of oral delivery, adenovirus vectors have previously demonstrated good tolerability and protective immunity against influenza virus in a phase II clinical trial [66]. Oral tablets have been applied for vaccine development of an adenovirus vector expressing the full-length SARS-CoV- $2 \mathrm{~S}$ and $\mathrm{N}$ proteins in combination with a Toll-like receptor-3 (TLR-3) agonist adjuvant [67]. Immunization of mice elicited strong neutralizing antibody responses, although in this case intranasal administration was preferred as the transgene expression in mice can be suppressed in the intestinal environment after oral administration [68]. However, the oral vaccine delivery approach has been evaluated in a phase I clinical trial for the VXA-COV2-1 vaccine candidate to assess safety and immunogenicity [69]. Preliminary data indicated that the vaccine was well tolerated and strong CD8 ${ }^{+} \mathrm{T}$-cell responses were induced [70]. Vaxart, the vaccine producer, claimed that the CD8 ${ }^{+}$T-cell responses obtained for VXA-COV2-1 were superior to those seen for the Pfizer/BioNTech and Moderna mRNA vaccines [71].

\section{SARS-CoV-2 variants and vaccine efficacy}

Despite the success achieved for developing vaccines against COVID-19 the detection of novel SARS-CoV-2 lineages has raised concern about vaccine efficacy. For instance, the B.1.1.7 variant (alpha) was initially claimed to possess higher transmission rates and was found to spread rapidly in the UK [72]. The alpha variant carrying the N510Y mutation and deletion of amino acids 69 and 70 in the RBD of the SARS-CoV-2 S protein was determined to be $75 \%$ more transmissible than the wildtype strain with the $501 \mathrm{~N}$ sequence [73]. Additionally, it was recently demonstrated that individuals tested positive for the alpha variant showed a 
mean $\log _{10}$ viral load 1.05 higher than non-alpha variant subjects [74]. In addition to the alpha variant, the South African B.1.351 (beta) [75], the Brazilian B.1.1.28.1 (gamma) [76], and the Indian B.1.617 (delta) [77] variants have been identified. Related to vaccine efficacy, adenovirus vector-, RNA-, and protein subunit-based vaccines have been tested. A small but significant reduction in neutralizing antibody activity against the N501A and the K417N-E484K-N501Y mutations in the SARS-CoV-2 S protein was detected for the two approved RNA-based vaccines [78]. In another study, 20 volunteers vaccinated with the BNT162b2 RNA vaccine showed similar neutralizing titers to SARS-CoV-2 with either N501 or Y501 in the S protein [79]. Related to the nanoparticle encapsulated SARS-CoV-2 S protein subunit vaccine NVX-CoV22373 the efficacy against the alpha variant was $86 \%$ and against the beta variant was $60 \%$ [80]. In the case of adenovirus-based vaccines, variability related to protection efficacy has been discovered. For instance, in a phase II/III trial, similar vaccine efficacy against the alpha variant and other lineages was obtained [15]. However, reduced neutralization activity was measured against the alpha variant compared to non-alpha variants in vitro after ChAfd0x1 $\mathrm{nCoV}-19$ vaccine administration [81]. Despite that, the vaccine provided protection against the alpha variant. However, in another study the ChAdOx1 nCoV-19 failed to provide protection against mild-to-moderated COVID-19 caused by the beta variant [82]. In contrast, the Ad26.COV2.S vaccine showed clinical efficacy against symptomatic COVID-19, also against the beta variant despite its partial resistance to neutralizing antibodies [83]. Moreover, humoral, and cellular responses against both the original SARS-CoV-2 strain and the beta variant were observed. However, the median pseudovirus neutralizing antibody titers were 5 -fold lower in comparison to the original SARS-CoV-2 strain. Overall, the detected variants and potentially emerging new variants demand a thorough follow-up on vaccine efficacy and the readiness of re-engineering available vaccines to ensure the efficacy of vaccine protection.

\section{Adenovirus vector-based vaccines and vaccine-induced immune thrombotic thrombocytopenia}

Recently, rare cases of thrombocytopenia have been detected in individuals receiving COVID-19 vaccines, which have been referred as VITT [84]. Although only a few hundred cases have been reported among the more than 150 million vaccinated people worldwide, the issue must be addressed properly. After the first detected cases in individuals vaccinated with the ChAdOx1 nCoV-2 vaccine, persons vaccinated with the Ad26. COV2.S vaccine also developed VITT [85]. As adenovirus gene transfer had previously been associated with VITT it was thought to have been induced by adenovirus administration [86]. However, not before long, cases of VITT were identified after vaccinations with the BNT162b2 and mRNA-1273 RNA vaccines [87]. One of the common factures for all vaccines causing VITT is the utilization of the SARS-CoV-2 S protein as the antigen. In a recent study, it was described that transcription of wildtype and codon-optimized SARS-CoV-2 S enables alternative splicing, which leads to production of C-terminal truncated soluble $\mathrm{S}$ protein variants [88]. It was postulated that the generated soluble $S$ protein variants are responsible for severe side effects by binding to ACE2 expressing endothelial cells in blood vessels leading to thromboembolic events. The disease mechanism was termed Vaccine-Induced COVID-19 Mimicry (VIC19M). Other hypotheses for the cause of VITT include the interaction of the SARS-CoV-2 S protein with C-type lectin receptors, heparin sulfate proteoglycans and the CD147 receptor, and interaction of the adenovirus vector with the CD46 receptor or platelet factor 4 antibodies [89]. Although some ideas and hypotheses have been presented, the reasons for causing VITT are still unresolved and requires further investigation.

\section{Conclusion}

Application of viral vectors for the development of COVID-19 has been extremely successful leading to EUA for several adenovirus-based vaccines in roughly one year since the onset of the pandemic. Although providing slightly lower vaccine efficacy than seen for RNA-based vaccines, they have been commonly used in mass vaccinations. However, comparison of efficacy of different COVID-19 vaccines is complicated. Although phase III clinical trials involve a large number of vaccinated individuals, the different vaccines are generally evaluated at different geographical locations and at different phases of the pandemic, and not compared in parallel. In this context, the adenovirus-based ChAdOx1 nCoV-19 and the mRNA-based BNT162b2 and mRMA-1273 vaccines were originally evaluated before the SARS-CoV- 2 alpha, beta, gamma, and delta variants 
emerged, which does not make the comparison to vaccines developed more recently accurate. However, several clinical trials are in progress for the current COVID-19 vaccines to determine vaccine efficacy against the latest SARS-CoV-2 variants.

One advantage of adenovirus-based vaccines compared to RNA-based vaccines is the superior storage and transportation requirements as these vaccines can be kept at $4^{\circ} \mathrm{C}$ and for shorter time at room temperature, whereas RNA-based vaccines require storage at $-20^{\circ} \mathrm{C}$ (Moderna) and $-80^{\circ} \mathrm{C}$ (Pfizer/BioNTech). Moreover, the Ad26.COV2.S has demonstrated efficacy after a single dose administration compared to the other vaccines requiring a prime-boost regimen. In addition to adenoviruses, vaccine candidates based on vectors of MV, vaccinia virus, rhabdoviruses, lentiviruses and influenza viruses have already been subjected to clinical trials. An interesting approach has been to employ self-amplifying RNA viruses for liposome nanoparticle-based delivery of mRNA. In this context, the VEE-based LNP-RNA vaccine candidate is currently under phase I clinical evaluation.

Although the current vaccines have demonstrated efficacy in humans, the occurrence of novel SARS-CoV-2 variants with enhanced transmissibility and pathogenicity have increased the demand on vaccine efficacy and the need for preparedness of re-engineering capacity of existent vaccines. Moreover, the findings of rare cases of VITT in individuals receiving COVID-19 vaccines require thorough investigations into the cause to be able to guarantee the highest possible safety for vaccine administration to prevent the spread of SARS-CoV-2 and to eradicate the pandemic.

\section{Abbreviations}

AAV: adeno-associated virus

ACE2: angiotensin-converting enzyme 2

ChAdV68: chimpanzee adenovirus serotype 68

COVID-19: coronavirus disease 2019

CTLs: cytotoxic T cell lymphocytes

DCs: dendritic cells

EUA: Emergency Use Authorization

FDA: Food and Drug Administration

GRAd: gorilla adenovirus

Ig: immunoglobulin

i.n.: intranasal

LNP: lipid nanoparticle

LV: lentivirus

LV-SMENP: lentivirus vector expressing severe acute respiratory syndrome coronavirus 2 minigene MV: measles virus

MVA: modified vaccinia virus Ankara

$\mathrm{N}$ : nucleocapsid

NDV: Newcastle disease virus

RBD: receptor binding domain

S: spike

SAM: self-amplifying mRNA

SARS-CoV-2: severe acute respiratory syndrome coronavirus 2

Th1: T helper 1

VEE: Venezuelan equine encephalitis virus

VITT: vaccine-induced immune thrombotic thrombocytopenia 
VSV: vesicular stomatitis virus

VSV- $\Delta \mathrm{G}$ : vesicular stomatitis virus $\mathrm{G}$ protein

\section{Declarations}

\section{Author contributions}

The author contributed solely to the work.

\section{Conflicts of interest}

The author declares that he has no conflicts of interest.

Ethical approval

Not applicable.

\section{Consent to participate}

Not applicable.

\section{Consent to publication}

Not applicable.

\section{Availability of data and materials}

Not applicable.

\section{Funding}

Not applicable.

\section{Copyright}

(c) The Author(s) 2021.

\section{References}

1. Izda V, Jeffries MA, Sawalha AH. COVID-19: a review of therapeutic strategies and vaccine candidates. Clin Immunol. 2021;222:108634.

2. Lundstrom K. Viral vectors for COVID-19 vaccine development. Viruses. 2021;13:317.

3. Lamb YN. BNT162b2 mRNA COVID-19 vaccine: first approval. Drugs. 2021;81:495-501.

4. Oliver SE, Gargano JW, Marin M, Wallace M, Curran KG, Chamberland M, et al. The advisory committee on immunization practices' interim recommendation for use of moderna COVID-19 vaccine - United States, December 2020. MMWR Morb Mortal Wkly Rep. 2021;69:1653-6.

5. Mallapaty S. WHO approval of Chinese CoronaVac vaccine will be crucial to curbing pandemic. Nature. 2021;594:161-2.

6. Keech C, Albert G, Cho I, Robertson A, Reed P, Neal S, et al. Phase 1-2 trial of a SARS-CoV-2 recombinant spike protein nanoparticle vaccine. N Engl J Med. 2020;383:2320-32.

7. Silveira MM, Moreira GMSG, Mendonça M. DNA vaccines against COVID-19: perspectives and challenges. Life Sci. 2021;267:118919.

8. Lundstrom K. Application of viral vectors for vaccine development with a special emphasis on COVID-19. Viruses. 2020;12:1324.

9. Harrison AG, Lin T, Wang P. Mechanisms of SARS-CoV-2 transmission and pathogenesis. Trends Immunol. 2020;41:1100-15.

10. Dormond E, Perrier M, Kamen A. From the first to the third generation adenoviral vector: what parameters are governing the production yield? Biotechnol Adv. 2009;27:133-44. 
11. van Doremalen N, Lambe T, Spencer A, Belij-Rammerstorfer S, Purushotham JN, Port JR, et al. ChAdOx1 nCov-19 vaccination prevents SARS-CoV-2 pneumonia in rhesus macaques. Nature. 2020;586:578-82.

12. Folegatti PM, Ewer KJ, Aley PK, Angus B, Becker S, Belij-Rammerstorfer S, et al. Safety and immunogenicity of the ChAdOx1 nCoV-19 vaccine against SARS-CoV-2: a preliminary report of a phase $1 / 2$ single-blind, randomised controlled trial. Lancet. 2020;396:467-78.

13. Ramasamy MN, Minassian AM, Ewer KJ, Flaxman AL, Folegatti PM, Owens DR, et al. Safety and immunogenicity of ChAdOx1 nCoV-19 vaccine administered in a prime-boost regimen in young and old adults (COV002): a single-blind, randomised, controlled, phase 2/3 trial. Lancet. 2021;396:1979-93.

14. Phase III double-blind, placebo-controlled study of AZD1222 for the prevention of COVID-19 in adults. ClinicalTrials.gov. [Internet]. [cited 2021 Jun 8]. Available from: https://clinicaltrials.gov/ct2/show/ NCT04516746

15. Voysey M, Costa Clemens SA, Madhi SA, Weckx LY, Folegatti PM, Aley PK, et al. Safety and efficacy of the ChAdOx1 nCoV-19 vaccine (AZD1222) against SARS-CoV-2: an interim analysis of four randomised controlled trials in Brazil, South Africa, and the UK. Lancet. 2021;397:99-111.

16. GOV.UK [Internet]. Regulatory approval of Vaxzevria (previously COVID-19 Vaccine AstraZeneca). c Crown copyright [cited 2021 Jun 8]. Available from: www.gov.uk/government/publications/ regulatory-approval-of-covid-19-vaccine-astrazeneca

17. Bos R, Rutten L, van der Lubbe JEM, Bakkers MJG, Hardenberg G, Wegmann F, et al. Ad26 vector-based COVID-19 vaccine encoding a prefusion-stabilized SARS-CoV-2 spike immunogen induces potent humoral and cellular immune responses. NPJ Vaccines. 2020;5:91.

18. Tostanoski LH, Wegmann F, Martinot J, Loos C, McMahan K, Mercado NB, et al. Ad26 vaccine protects against SARS-CoV-2 severe clinical disease in hamsters. Nat Med. 2020;26:1694-1700.

19. Mercado NB, Zahn R, Wegmann F, Loos C, Chandrashekar A, Yu J, et al. Single-shot Ad26 vaccine protects against SARS-CoV-2 in rhesus macaques. Nature. 2020;586:583-8.

20. Stephenson KE, Le Gars M, Sadoff J, de Groot AM, Heerwegh D, Truyers C, et al. Immunogenicity of the Ad26.COV2.S vaccine for COVID-19. JAMA. 2021;325:1535-44.

21. Sadoff J, Le Gars M, Shukarev G, Heerwegh D, Truyers C, de Groot AM, et al. Interim results of a phase 1-2a trial of Ad26.COV.S Covid-19 vaccine. N Engl J Med. 2021;384:1824-35.

22. A study of Ad26.COV2.S for the prevention of SARS-CoV-2-mediated COVID-19 in adult participants (ENSEMBLE). ClinicalTrials.gov. [Internet]. [cited 2021 Jun 8]. Available from: https://clinicaltrials.gov/ ct2/show/NCT04505722

23. drugs.com [Internet]. Janssen COVID-19 vaccine FDA approval status. c2000-2021 [cited 2021 Jun 10]. Available from: https://www.drugs.com/history/janssen-covid-19-vaccine.html

24. clinicaltrialsarena.com [Internet]. Russia reports positive early data on Covid-19 vaccine Sputnik V. c2021 [cited 2021 Jun 10]. Available from: www.clinicaltrialsarena.com

25. Logunov DY, Dolzhikova IV, Zubkova OV, Tukhvatullin AI, Shcheblyakov DV, Dzharullaeva AS, et al. Safety and immunogenicity of an rAd 26 and rAd5 vector-based heterologous prime-boost COVID-19 vaccine in two formulations: two open, non-randomised phase 1/2 studies from Russia. Lancet. 2020;396:887-97.

26. Logunov DY, Dolzhikova IV, Shcheblyakov DV, Tukhavatulin AY, Zubkova OV, Dzharullaeva AS, et al. Safety and efficacy of an rAd26 and rAd5 vector-based heterologous prime-boost COVID-19: an interim analysis of a randomised controlled phase 3 in Russia. Lancet. 2021;397:671-81.

27. Callaway E. Russia's fast-track coronavirus vaccine draws outrage over safety. Nature. 2020;584:334-5.

28. Wu S, Zhong G, Zhang J, Shuai L, Zhang Z, Wen Z, et al. A single dose of an adenovirus-vectored vaccine provides protection against SARS-CoV-2 challenge. Nat Commun. 2020;11:4081.

29. Feng L, Wang $Q$, Shan $C$, Yang $C$, Feng $Y$, Wu J, et al. An adenovirus-vectored COVID-19 vaccine confers protection from SARS-CoV-2 challenge in rhesus macaques. Nat Commun. 2020;11:4207. 
30. Zhu FC, Li YH, Guan XH, Hou LH, Wang WJ, Li JX, et al. Safety, tolerability, and immunogenicity of a recombinant adenovirus type-5 vectored COVID-19 vaccine: a dose-escalation, open-label, non-randomised, first-in-human trial. Lancet. 2020;395:1845-54.

31. Zhu FC, Guan XH, Li YH, Huang, JY, Jiang T, Hou LH, et al. Immunogenicity and safety of a recombinant adenovirus type-5-vectored COVID-19 vaccine in healthy adults aged 18 years or older: a randomised, double-blind, placebo-controlled, phase 2 trial. Lancet. 2020;396:479-88.

32. cansinotech.com [Internet]. NMPA accepts the application for conditional marketing authorization of CanSinoBIO's COVID-19 vaccine Convidecia ${ }^{\mathrm{TM}}$. c2017 [cited 2021 Jun 11]. Available from: http://www. cansinotech.com/html/1//179/180/651.html

33. reuters.com [Internet]. China approves two more domestic COVID-19 vaccines for public use. c2021 [cited 2021 Jun 11]. Available from: www.reuters.com/article/us-health-coronavirus-china-vaccineidUSKBN2AP1MW

34. Capone S, Raggioli A, Gentile M, Battella S, Lahm A, Sommella A, et al. Immunogenicity of a new gorilla adenovirus vaccine candidate for COVID-19. Mol Ther. 2021;2412:23.

35. GRAd-COV2 vaccine against COVID-19. ClinicalTrials.gov. [Internet]. [cited 2021 Jun 11]. Available from: https://clinicaltrials.gov/ct2/show/NCT04528641

36. Roshorm Y, Cottingham MG, Potash MJ, Volsky DJ, Hanke T. T cells induced by recombinant chimpanzee adenovirus alone and in prime-boost regimens decrease chimeric EcoHIV/NDK challenge virus load. Eur J Immunol. 2012;42:3243-55.

37. Chimpanzee adenovirus and self-amplifying mRNA prime-boost prophylactic vaccines against SARS-CoV-2 in healthy adults. ClinicalTrials.gov. [Internet]. [cited 2021 Jun 11]. Available from: https:// clinicaltrials.gov/ct2/show/NCT04776317

38. Zabaleta N, Dai W, Bhatt U, Hérate C, Maisonnasse P, Chichester JA, et al. An AAV-based, room-temperature-stable, single dose COVID-19 vaccine provides durable immunogenicity and protection in non-human primates. Cell Host Microbe. 2021;29:1437-53.

39. Kreijtz JH, Suezer Y, van Amerongen G, de Mutsert G, Schnierle BS, Wood JM, et al. Recombinant modified vaccinia virus Ankara-based vaccine induces protective immunity in mice against infection with influenza virus H5N1. J Infect Dis. 2007;195:1598-1606.

40. Chiuppesi F, Salazar MD, Contreras H, Nguyen VH, Martinez J, Park Y, et al. Development of a multi-antigenic SARS-CoV-2 vaccine candidate using a synthetic poxvirus platform. Nat Commun. 2020;11:6021.

41. García-Arriaza J, Garaigorta U, Pérez P, Lázaro-Frías A, Zamora C, Gastaminza P, et al. COVID-19 vaccine candidates based on modified vaccinia Ankara expressing the SARS-CoV-2 spike induce robust T- and B-cell immune responses and fully efficacy in mice. J Virol. 2021;95:e2260-20.

42. Safety, tolerability and immunogenicity of the candidate vaccine MVA-SARS-2-S against COVID-19. ClinicalTrials.gov. [Internet]. [cited 2021 Jun 11]. Available from: https://clinicaltrials.gov/ct2/show/ NCT04569383

43. A synthetic MVA-based SARS-CoV-2 vaccine, COH04S1, for the prevention of COVID-19. ClinicalTrials. gov. [Internet]. [cited 2021 Jun 11]. Available from: https://clinicaltrials.gov/ct2/show/NCT04639466

44. Burman B, Pesci G, Zamarin D. Newcastle disease virus at the forefront of cancer immunotherapy. Cancers. 2020;12:3552.

45. Sun W, McCroskery S, Liu WC, Leist SR, Liu Y, Albrecht RA, et al. A Newcastle disease virus (NDV) expressing a membrane-anchored spike as a cost-effective inactivated SARS-CoV-2 vaccine. Vaccines. 2020;8:771.

46. Sun W, Leist SR, McCroskery S, Liu Y, Slamanig S, Oliva J, et al. Newcastle disease virus (NDV) expressing the spike protein of SARS-CoV-2 as a live virus vaccine candidate. EBioMedicine. 2020;62:103132.

47. Assess the safety and immunogenicity of NDV-HXP-S vaccine in Thailand. ClinicalTrials.gov. [Internet]. [cited 2021 Jun 11]. Available from: https://clinicaltrials.gov/ct2/show/NCT04764422 
48. Lundstrom K. Self-amplifying RNA viruses as RNA vaccines. Intl J Mol Sci. 2020;21:5130.

49. Hörner C, Schürmann C, Auste A, Ebenig A, Muraleedharan S, Dinnon KH 3rd, et al. A highly immunogenic and effective measles virus-based Th1-biased COVID-19 vaccine. Proc Natl Acad Sci U S A. 2020;117:32657-66.

50. Clinical trial to evaluate the safety and immunogenicity of the COVID-19 vaccine (COVID-19-101). ClinicalTrials.gov. [Internet]. [cited 2021 Jun 11]. Available from: https://clinicaltrials.gov/ct2/show/ NCT04497298

51. merck.com [Internet]. Merck discontinues development of SARS-CoV-2/COVID-19 vaccine candidates; continues development of two investigational therapeutic candidates. c2009-2021 [cited 2021 Jun 11]. Available from: http://www.merck.com/news/merck-discontinues-development-of-sars-cov-2-covid19-vaccine-candidates-continues-development-of-two-investigational-therapeutic-candidates/

52. Case JB, Rothlauf PW, Chen RE, Kafai NM, Fox JM, Smith BK, et al. Replication-competent vesicular stomatitis virus vaccine vector protects against SARS-CoV-2-mediated pathogenesis in mice. Cell Host Microbe. 2020;28:465-74.e4.

53. Dose ranging trial to assess safety and immunogenicity of V590 (COVID-19 vaccine) in healthy adults (V590-001). ClinicalTrials.gov. [Internet]. [cited 2021 Jun 11]. Available from: https://clinicaltrials.gov/ ct2/show/NCT04569786

54. iavi.org [Internet]. Merck and IAVI discontinue development of COVID-19 vaccine candidate V590. c2021 [cited 2021 Jun 11]. Available from: https://www.iavi.org/news-resources/press-releases/2021/ merck-and-iavi-discontinue-development-of-covid-19-vaccine-candidate-v590

55. Yahalom-Ronen Y, Tamir H, Melamed S, Politi B, Shifman O, Achdout H, et al. A single dose of recombinant VSV- $\Delta$ G-spike provides protection against SARS-CoV-2 challenge. Nat Commun. 2020;11:6402.

56. Evaluate the safety, immunogenicity and potential efficacy of an rVSV-SARS-CoV-2-S vaccine. ClinicalTrials.gov. [Internet]. [cited 2021 Jun 11]. Available from: https://clinicaltrials.gov/ct2/show/ NCT04608305

57. Levin Y, Balakirski NM, Caraco Y, Ben-Ami E, Atsmon J, Marcus H. Ethics and execution of developing a 2nd wave COVID vaccine-our interim phase I/II VSV-SARS-CoV2 vaccine experience. Vaccine. 2021;39:2821-3.

58. McKay PF, Hu K, Blakney AK, Samnuan K, Brown JC, Penn R, et al. Self-amplifying RNA SARS-CoV-2 lipid nanoparticle vaccine candidate induces high neutralizing antibodies in mice. Nat Commun. 2020;11:3523.

59. Isrctn.com [Internet]. Clinical trial to assess the safety of a coronavirus vaccine in healthy men and women. c2021 [cited 2021 Jun 14]. Available from: http://www.isrctn.com/ISRCTN17072692

60. King RG, Silva-Sanchez A, Peel JN, Botta D, Meza Perez S, Allie SR, et al. Single-dose intranasal administration of AdCOVID elicits systemic and mucosal immunity against SARS-CoV-2 in mice. bioRxiv 331348 [Preprint]. 2020 [cited 2021 Jun 11]. Available from: https://www.biorxiv.org/ content/10.1101/2020.10.10.331348v1

61. Ku MW, Bourgine M, Authie P, Lopez J, Nemirov K, Moncoq F, et al. Intranasal vaccination with a lentiviral vector protects against SARS-CoV-2 in preclinical animal models. Cell Host Microbe. 2021;29:236-49.

62. Immunity and safety of Covid-19 synthetic minigene vaccine. ClinicalTrials.gov. [Internet]. [cited 2021 Jun 11]. Available from: https://clinicaltrials.gov/ct2/show/NCT04276896

63. Loes AN, Gentles LE, Greaney AJ, Crawford KHD, Bloom JD. Attenuated influenza virions expressing the SARS-CoV-2 receptor-binding domain induce neutralizing antibodies in mice. Viruses. 2020;12:987.

64. chictr.org [Internet]. A phase I clinical trial of influenza virus vector COVID-19 vaccine for intranasal spray (DelNS1-2019-nCoV-RBD-OPT1). ChiCTR2000037782. [cited 2021 Jun 14]. Available online: http://www.chictr.org.cn/showprojen.aspx?proj=55421 
65. chictr.org [Internet]. A phase II clinical trial of influenza virus vector COVID-19 vaccine for intranasal spray (DelNS1-2019-nCoV-RBD-OPT1). ChiCTR2000039715. [cited 2021 Jun 14]. Available online: http://www.chictr.org.cn/showprojen.aspx?proj=63754

66. Liebowitz D, Gottlieb K, Kolhatkar NS, Garg SJ, Asher JM, Nazareno J, et al. Efficacy, immunogenicity, and safety of an oral influenza vaccine: a placebo-controlled and active-controlled phase 2 human challenge study. Lancet Infect Dis. 2020;20:435-44.

67. Moore AC, Dora EG, Peinovich N, Tucker KP, Lin K, Cortese M, et al. Pre-clinical studies of a recombinant adenoviral mucosal vaccine to prevent SARS-CoV-2 infection. bioRxiv 283853 [Preprint]. 2020 [cited 2021 Jun 11]. Available from: https://www.biorxiv.org/content/10.1101/2020.09.04.283853v1

68. Revaud J, Unterfinger Y, Rol N, Suleman, M, Shaw J, Galea S, et al. Firewalls prevent systemic dissemination of vectors derived from human adenovirus type 5 and suppress production of transgene-encoded antigen in a murine model of oral vaccination. Front Cell Infect Microbiol. 2018;8:6.

69. Safety and immunogenicity trial of an oral SARS-CoV-2 vaccine (VXA-CoV2-1) for prevention of COVID-19 in healthy adults. ClinicalTrials.gov. [Internet]. [cited 2021 Jun 11]. Available from: https://clinicaltrials. gov/ct2/show/NCT04563702

70. vaxart.com [Internet]. Vaxart announces positive preliminary data from phase 1 clinical trial evaluating its oral COVID-19 tablet vaccine candidate. c2021 [cited 2021 Jun 16]. Available from: https://investors. vaxart.com/news-releases/news-release-details/vaxart-announces-positive-preliminary-data-phase1-clinical

71. vaxart.com [Internet]. New data from Vaxart oral COVID-19 vaccine phase I study suggests broad cross-reactivity against other coronaviruses. c2021 [cited 2021 Jun 16]. Available from: https:// investors.vaxart.com/news-releases/news-release-details/new-data-vaxart-oral-covid-19-vaccinephase-i-study-suggests

72. Leung K, Shum MH, Leung GM, Lam TT, Wu JT. Early transmissibility assessment of the N501Y mutant strains of SARS-CoV-2 in the United Kingdom, October to November 2020. Euro Surveill.2021;26:2002106.

73. Galloway SE, Paul P, MacCannell DR, Johansson MA, Brooks JT, MacNeil A, et al. Emergence of SARS-CoV-2 B.1.1.7 lineage-United States, 29 December 2020-12 January 2021. MMWR Morb Mortal Wkly Rep. 2021;70:95-9.

74. Jones TC, Biele G, Mühlemann B, Veith T, Schneider J, Beheim-Schwarzbach, et al. Estimating infectiousness throughout SARS-CoV-2 infection course. Science. 2021;373:eabi5273.

75. Mwenda M, Saasa N, Sinyange N, Busby G, Chipimo PJ, Hendry J, et al. Detection of B.1.351 SARS-CoV-2 variant strain—Zambia, December 2020. MMWR Morb Mortal Wkly Rep. 2021;70:280-2.

76. Das JK, Sengupta A, Choudhury PP, Roy S. Characterizing genomic variants and mutations in SARS-CoV-2 proteins from Indian isolates. Gene Rep. 2021;[Epub ahead of print].

77. Cantón R, De Lucas Ramos P, García-Botella A, AGarcía-Lledó A, Gómez-Pavón J, González Del Castillo J, et al. New variants of SARS-CoV-2. Rev Esp Quimiother. 2021; [Epub ahead of print].

78. Wang Z, Schmidt F, Weisblum Y, Muecksch F, Barnes CO, Finkin S, et al. mRNA vaccine-elicited antibodies to SARS-CoV-2 and circulating variants. Nature. 2021;592:616-22.

79. Xie X, Zou J, Fontes-Garfias CR, Xia H, Swanson KA, Cutler M, Cooper D, et al. Neutralization of N501Y mutant SARS-CoV-2 by BNT162b vaccine-elicited sera. bioRxiv 425740 [Preprint]. 2021 [cited 2021 Jun 11]. Available from: https://www.biorxiv.org/content/10.1101/2021.01.07.425740v1

80. Mahase E. Covid-19: Novavax vaccine efficacy is $86 \%$ against UK variant and $60 \%$ against South African variant. BMJ. 2021;372:n296.

81. Emary KRW, Golubchik T, Aley PK, Ariani CV, Angus B, Bibi S, et al. Efficacy of ChAdOx1 nCoV-19 (AZD1222) vaccine against SARS-CoV-2 variant of concern 202012/01 (B.1.1.7): an exploratory analysis of a randomised controlled trial. Lancet. 2021;397:1351-62. 
82. Madhi SA, Baillie V, Cutland, CL, Voysey M, Koen AL, Fairlie SD, et al. Efficacy of the ChAdOx1 nCoV-19 Covid-19 vaccine against the B.1.351 variant. N Engl J Med. 2021;1885-98.

83. Alter G, Yu J, Liu J, Chandrashekar A, Borducchi EN, Tostanoski LH, et al. Immunogenicity of Ad26.COV2.S vaccine against SARS-CoV-2 variants in humans. Nature. 2021;596:268-72.

84. Greinacher A, Thiele T, Warkentin TE, Weisser K, Kyrle PA, Eichinger S. Thrombotic thrombocytopenia after ChAdOx1 nCoV-19 vaccination. N Engl J Med. 2021;384:2092-2101.

85. Muir KL, Kallam A, Koepsell SA, Gundabolu K et al. Thrombotic thrombocytopenia after Ad26.COV.S vaccination. N Engl J Med. 2021; 1964-65.

86. Othman M, Labelle A, Mazzetti I, Elbatarny HS, Lillicrap D. Adenovirus-induced thrombocytopenia: the role of von Willebrand factor and P-selectin in mediating accelerated platelet clearance. Blood. 2007;109:2832-9.

87. Lee EJ, Cines DB, Gernsheimer T, Kessler C, Michel M, Tranatino MD, et al. Thrombocytopenia following Pfizer and Moderna SARS-CoV-2 vaccination. Am J Hematol. 2021;534-7.

88. Kowarz E, Krutzke L, Reis J, Bracharz S, Kochanek S, Marschalek R. "Vaccine-induced Covid-19 mimicry" syndrome: splice reactions within the SARS-CoV-2 spike open reading frame result in spike protein variants that may cause thromboembolic events in patients immunized with vector-based vaccines. Res Square. 2021;[Epub ahead of print].

89. Lundstrom K, Barh D, Uhal BD, Takayama K, Aljabali AAA, Mohamed Abd El-Aziz T, et al. CoVID-19 vaccines and thrombosis-roadblock or dead-end street? Biomolecules. 2021;11:1020. 